\title{
RESEARCH
}

Open Access

\section{Optic nerve thinning and neurosensory retinal degeneration in the rTg4510 mouse model of frontotemporal dementia}

\author{
Ian F. Harrison ${ }^{1 *}\left(\mathbb{D}\right.$, Rozalind Whitaker ${ }^{2}$, Pietro Maria Bertellii, ${ }^{2,4}$, James M. O'Callaghan ${ }^{1}$, Lajos Csincsik ${ }^{2,4}$,
} Martina Bocchetta ${ }^{3}$, Da Ma $^{1,5}$, Alice Fisher ${ }^{6}$, Zeshan Ahmed ${ }^{6}$, Tracey K. Murray ${ }^{6}$, Michael J. O'Neill ${ }^{6}$, Jonathan D. Rohrer ${ }^{3}$, Mark F. Lythgoe ${ }^{1}$ and Imre Lengyel ${ }^{2,4}$

\begin{abstract}
Visual impairments, such as difficulties in reading and finding objects, perceiving depth and structure from motion, and impaired stereopsis, have been reported in tauopathy disorders, such as frontotemporal dementia (FTD). These impairments however have been previously attributed to cortical pathologies rather than changes in the neurosensory retina or the optic nerve. Here, we examined tau pathology in the neurosensory retina of the $r \operatorname{Tg}(\operatorname{tauP301L)4510}$ mouse model of FTD. Optic nerve pathology in mice was also assessed using MRI, and corresponding measurements taken in a cohort of five FTD sufferers and five healthy controls. rTg(tauP301L)4510 mice were imaged (T2-weighted MRI) prior to being terminally anesthetized and eyes and brains removed for immunohistochemical and histological analysis. Central and peripheral retinal labelling of tau and phosphorylated tau (pTau) was quantified and retinal layer thicknesses and cell numbers assessed. MR volumetric changes of specific brain regions and the optic nerve were compared to tau accumulation and cell loss in the visual pathway. In addition, the optic nerves of a cohort of healthy controls and behavioural variant FTD patients, were segmented from T1- and T2-weighted images for volumetric study. Accumulation of tau and pTau were observed in both the central and peripheral retinal ganglion cell (RGC), inner plexiform and inner nuclear layers of the neurosensory retina of rTg(tauP301L)4510 mice. This pathology was associated with reduced nuclear density $(-24.9 \pm 3.4 \%)$ of the central RGC layer, and a reduced volume $(-19.3 \pm 4.6 \%)$ and elevated T2 signal $(+27.1 \pm 1.8 \%)$ in the optic nerve of the transgenic mice. Significant atrophy of the cortex (containing the visual cortex) was observed but not in other area associated with visual processing, e.g. the lateral geniculate nucleus or superior colliculus. Atrophic changes in optic nerve volume were similarly observed in FTD patients $(-36.6 \pm 2.6 \%)$. The association between tau-induced changes in the neurosensory retina and reduced optic nerve volume in mice, combined with the observation of optic nerve atrophy in clinical FTD suggests that ophthalmic tau pathology may also exist in the eyes of FTD patients. If tau pathology and neurodegeneration in the retina were to reflect the degree of cortical tau burden, then cost-effective and non-invasive imaging of the neurosensory retina could provide valuable biomarkers in tauopathy. Further work should aim to validate whether these observations are fully translatable to a clinical scenario, which would recommend follow-up retinal and optic nerve examination in FTD.
\end{abstract}

Keywords: Tau, Frontotemporal dementia, Neurosensory retina, MRI

\footnotetext{
* Correspondence: lan.harrison@ucl.ac.uk

Ian F. Harrison and Rozalind Whitaker are joint first authors

Mark F. Lythgoe and Imre Lengyel are joint senior authors

${ }^{1}$ UCL Centre for Advanced Biomedical Imaging, Division of Medicine,

University College London, Paul O'Gorman Building, 72 Huntley Street,

London WC1E 6DD, UK

Full list of author information is available at the end of the article
}

(c) The Author(s). 2019 Open Access This article is distributed under the terms of the Creative Commons Attribution 4.0 International License (http://creativecommons.org/licenses/by/4.0/), which permits unrestricted use, distribution, and reproduction in any medium, provided you give appropriate credit to the original author(s) and the source, provide a link to the Creative Commons license, and indicate if changes were made. The Creative Commons Public Domain Dedication waiver (http://creativecommons.org/publicdomain/zero/1.0/) applies to the data made available in this article, unless otherwise stated. 


\section{Introduction}

Frontotemporal dementia (FTD) is a neurodegenerative disorder characterised by degeneration within the frontal and temporal lobes of the brain [20, 41, 46]. The disorder presents clinically with changes in behaviour, deficits in executive function and/or language impairment, but in some cases can also manifest with a motor disorder such as corticobasal syndrome, motor neuron disease or progressive supranuclear palsy (PSP) [44]. Around 30-50\% of FTD cases are familial in nature, with an autosomal dominant profile of inheritance [44], and in a subset of these cases, causative mutations in the microtubule associated protein tau (MAPT) gene have been identified [16]. Such mutations lead to disruption of the normal binding of tau to neuronal tubulin, resulting in pathological deposition of hyperphosphorylated tau in the form of filamentous neuronal inclusions, or neurofibrillary tangles (NFTs) [16, 39]. NFTs are also observed in other pathologies such as Alzheimer's disease (AD), Pick's disease, corticobasal degeneration and PSP, a group of neurological disorders collectively known as tauopathies.

Numerous reports exist of early stage tauopathy patients experiencing cognitive visual changes, including changes in colour recognition [9], impairment of spatial contrast sensitivity [15], depth perception [28], perceiving structure from motion [10, 28], and also difficulties with reading and finding objects [21]. Previously however, these defects have been attributable to the well described cortical tau pathology in the brains of patients with tauopathies rather than the result of tau pathology in the optic nerve or neurosensory retina. Responsible for converting incoming light into encoded neural activity, the neurosensory retina is a laminal structure of nuclear and neuropil plexiform layers, formed by a tripartite neuronal relay: Photoreceptors - > Bipolar cells - > Retinal Ganglion Cells (RGCs), which is modulated by two classes of interneurons, Horizontal and Amacrine cells, and supported by Müller glia. RGC axons converge in the Retinal Nerve Fibre Layer (RNFL) to form the optic nerve, which then projects to the visual centres of the brain for higher order processing of input signals. With advancing age, tau becomes expressed diffusely within the inner nuclear layer (INL), and the retinal ganglion cell layer (RGCL) of the human neurosensory retina [24]. Furthermore in ocular diseases such as glaucoma, abnormally phosphorylated tau is increased in the INL and inner plexiform layer (IPL) of the retina $[17,31,48]$, an observation which has similarly been made in a mouse model of ocular hypertension induced glaucoma [8]. Based on this evidence, that tau pathology also affects the eye, we hypothesized that retinal and optic nerve changes might also be associated with the pathologies in tauopathic dementias such as FTD.

Assessments of retinal changes in mice expressing the P301S mutated human MAPT gene driven by the mouse
Thy1.2 promoter (mThy1.2) showed increased phosphorylation of tau in the RGCL and filamentous inclusions in the retina [14] without an effect on RGC number. In transgenic mice expressing the P301L mutation in the human MAPT gene however, driven by the mouse prion promoter $(P R N P)$, total tau and phosphorylated tau levels were shown to be elevated in the RGCL, the IPL and also the INL, accompanied by a reduction in the thickness of the INL, an effect which was more pronounced in the peripheral compared to the central retina [18]. These changes were accompanied by an increase in the number but reduction in the average size of RGCs in the transgenic mice [18]. Together these data highlight the susceptibility of the neurosensory retina to mutations associated with familial FTD, and hence highlights the possibility of retinal changes in the clinical scenario. However both of these transgenic models (MAPT P301S driven by mThy1.2 [14], and MAPT P301L driven by PRNP [18]) show ubiquitous expression of pathological phosphorylated tau throughout the brain, quite unlike the pathological spatial pattern of tau observed in FTD [20, $41,46]$. Therefore, in order to better understand the effects of mutations associated with familial FTD on the neurosensory retina and visual system, we studied an animal model that shows spatial patterns of tau deposition similar to that seen in clinical FTD.

In the current study we assessed retinal tau pathology in the $\operatorname{rTg}(\operatorname{tau} 301 \mathrm{~L}) 4510$ mouse model, which harbours the same P301L mutant form of the human MAPT gene previously described in the study by Ho et al. [19], but driven by the forebrain-specific calmodulin kinase II a (CaMKIIa) promoter system, which results in NFT pathology in forebrain structures and the hippocampus, reminiscent of the pattern for tau accumulation observed in FTD [20, 41, 46]. Using this animal model, we show increased phosphorylated tau expression in the RGCL, as well as a decrease in RGC numbers in the neurosensory retina associated with volumetric changes (assessed through MRI) in the optic nerve and areas of the mouse brain involved in visual processing. In addition, a cohort of FTD patients carrying the $M A P T$ mutation were imaged with MRI, and we show that similar to mice, volumetric changes in the optic nerve do take place in familial FTD.

\section{Materials and methods \\ Animals}

Generation of homozygous rTg(tauP301L)4510 transgenic mice, henceforth referred to as rTg4510 mice, has been described previously [43]. The rTg4510 and litter matched wildtype mice were bred on a mixed $\mathrm{FVB} / \mathrm{NCrl}$ +129 S6/SvEvTa background for Eli Lilly by Taconic (Germantown, Maryland, USA), licensed from the Mayo Clinic (Jacksonville, Florida, USA), and imported into the United Kingdom for study at the UCL Centre for 
Advanced Biomedical Imaging. Mice were kept in individually ventilated cages in groups of $3-5$, with ad libitum access to food and water. Animal weight was monitored upon arrival and until experimentation, and all animals were deemed healthy prior to experiments.

For this study, import of 10 female 7.5 month of age rTg4510 and 10 female litter matched wildtype mice occurred two weeks prior to initial imaging studies and subsequent sacrifice, perfuse fixation, and sectioning for immunofluorescent analysis. All the experiments were performed in accordance with the Animals (Scientific Procedures) Act 1986 (ASPA) revised according to the European Directive 2010/63/EU and the UK Home Office (Scientific Procedures) Act (1986) with prior project approval from UCL's internal Animal Welfare and Ethical Review Body.

\section{Magnetic resonance imaging of mice Image acquisition}

All imaging was performed with a 9.4 T VNMRS horizontal bore scanner (Agilent Inc). A $72 \mathrm{~mm}$ inner diameter volume coil (Rapid Biomedical) was used for radiofrequency transmission, and signal was received using a 4-channel array head coil (Rapid Biomedical). Mice were placed in an induction box before anaesthesia was induced using $2 \%$ isoflurane at $1 \mathrm{~L} / \mathrm{min}$ in $100 \% \mathrm{O}_{2}$. Mice were subsequently positioned in an MRI-compatible head holder to minimize motion artefacts. Anaesthesia was maintained throughout imaging using $1.5 \%( \pm 0.2 \%)$ isoflurane at $1 \mathrm{~L} / \mathrm{min}$ in $100 \% \mathrm{O}_{2}$ delivered via a nose cone, which permitted spontaneous breathing of the mice. Core temperature and respiratory rate were monitored using a rectal probe and pressure pad, respectively (SA Instruments). Mice were maintained at $\sim 37^{\circ} \mathrm{C}$ using heated water tubing and a warm air blower with a feedback system (SA Instruments). Respiration rate was maintained between 80 and 120 breaths per minute by manually adjusting the isoflurane vaporizer.

A 3-dimensional T2-weighted fast spin echo sequence was employed for structural imaging with the following parameters: field of view $(\mathrm{FOV})=19.2 \times 16.8 \times 12.0 \mathrm{~mm}$, resolution $=150 \times 150 \times 150 \mu \mathrm{m}$, repetition time $(\mathrm{TR})=$ $2500 \mathrm{~ms}$, effective echo time $=43 \mathrm{~ms}$, echo train length $=$ 4 , number of signal averages $=1$, and imaging time $=1.5 \mathrm{~h}$.

Mice were imaged in a random order, working through cages which housed a mixture of $\operatorname{rTg} 4510$ and litter matched wildtype mice.

\section{Image analysis}

For manual segmentation of the optic nerve and eye structures for extraction of volumes and signal intensities on T2-weighted images, ITK-SNAP segmentation software (v3.x) [49] was used. For extraction of brain volumes, a multi-atlas-based structural parcellation framework was used [25]. Using this framework, we extracted 4 structures of interest to the visual system and dementia: the cortex (containing the visual cortex), thalamus (containing the lateral geniculate nucleus), hippocampus, and superior colliculus. The lateral geniculate nucleus and superior colliculus were of interest as in both humans and mice they are key targets of optic tract axons, and contribute to visual processing, as well as acting as a relay to other destinations in the visual pathway. In addition, we extracted the volume of the whole brain for normalisation purposes. For this, brain images were oriented, non-uniformity corrected, and skull stripped. We adopted the publicly available in vivo mouse brain MRI atlas previously published for the framework [26]. First, the atlas images were registered affinely to the original MR image data using a block-matching algorithm [36]. Once complete, the STAPLE algorithm [25] was applied to fuse the resampled atlas masks together to create a consensus brain mask for each animal's scans. A further non-rigid registration based on fast free-form deformation was then performed to correct any remaining local misalignment of the affinely registered atlas to the brain volumes [30]. The structural labels from the atlas were then transformed and resampled to the image space of the brain scans using the same affine and non-rigid transformation and fused using the STEPS label fusion algorithm [22] to create the final parcellated structures of interest: the cortex, thalamus, hippocampus, and superior colliculus. A previously published calibration protocol was used to correct gradient scaling errors in the data [32]. Both absolute volumes and volumes normalised to the extracted whole brain volume are presented.

\section{CSF extraction, perfuse fixation and tissue preparation for histology}

Following in vivo MR imaging, animals were terminally anaesthetised with an overdose of Euthanal administered via intraperitoneal injection. A midline incision was made at a midpoint between the skull base and the occipital margin to the first vertebrae. The underlying muscles were parted to expose the atlanto-occipital membrane and dura mater overlaying the cisterna magna. The area was cleaned and a durotomy performed with a 23-gauge needle, allowing CSF to be collected using a narrow bore pipette tip. The thoracic cavities were then opened and the animals were intracardially perfused through the left ventricle: first with $15-20 \mathrm{~mL}$ of saline $(0.9 \%)$ and heparin; second with $50 \mathrm{~mL}$ of Formalin, at a flow rate of $3 \mathrm{~mL}$ per minute. Following perfusion, the animal was decapitated, de-fleshed, and the head stored at $4{ }^{\circ} \mathrm{C}$ and soaked in Formalin for 9 weeks. Brains were removed from the skull and processed using the Tissue TEK VIP processor (GMI Inc.) and embedded in paraffin wax. $6 \mu \mathrm{m}$ thick sections of the brain in the sagittal plane were collected using a 
rotary microtome and mounted on glass slides for immunohistochemistry. The eye globes were enucleated, processed (Leica ASP3005) and embedded in paraffin in an orientation that facilitated sectioning in transverse planes. Ice cooled paraffin tissue blocks were sectioned (Leica RM2235 Microtome using S35-PFM feather microtome blades) to generate $4 \mu \mathrm{m}$ sections which were then placed in a $45^{\circ} \mathrm{C}$ water bath. Once mounted onto Super-Frost Plus slides, sections were drip-dried for 2-5 min before heat fixing for 60 mins. Haematoxylin and Eosin (H\&E) staining (Autostainer Leica CV5030) and light microscopy (Olympus multi-head light microscope with Micropixx camera software) enabled semi-quantitative morphometric analysis (Image J) and identification of each sections anatomical location in the retinal peripheral-central axis. Sections within $25 \mu \mathrm{m}$ of the optic nerve head were subsequently used for immunohistochemical staining. The central and peripheral retina were defined as the most lateral and central non-fragmented region of each tissue section (Additional file 1: Figure S1). H\&E derived semi-quantitative data included Retinal Ganglion Cell Layer (RGCL) nuclear densities and Inner Nuclear Layer (INL) nuclear densities (number of nuclei occupying a measured area of RGCL or INL respectively), and Inner Plexiform Layer (IPL) thickness relative to total retinal thickness. This data was generated for peripheral and central retinal regions.

\section{Quantification of CSF tau}

The collected CSF was centrifuged briefly to collect any red blood cell contaminates, the supernatant removed and frozen at $-20^{\circ} \mathrm{C}$ until further analysis by ELISA. $2 \mu \mathrm{l}$ water was added to the blood pellet and snap frozen on dry ice to ensure hypotonic freeze-thaw release of haemoglobin from any red blood cells present. Each sample was measured at $417 \mathrm{~nm}$ on a NanoDrop spectrophotometer to quantify percentage blood contamination of each CSF sample. This method allows for measurement of blood contamination down to $0.0001 \%$, well below that detectable by eye $(\sim 0.01 \%)$. Lack of significant blood contamination was verified by an average percentage contamination of $0.003 \%( \pm 0.0006 \%)$. Tau content of CSF samples were quantified using both the Human Tau (total), and Human pTau [pS ${ }_{199}$ ] ELISA Kits (Invitrogen, UK) as per the manufacturer's instructions.

\section{Immunohistochemistry}

For immunohistochemistry in the retina, retinal sections from $5 \mathrm{rTg} 4510$ and 4 wildtype mice were deparaffinised and hydrated by sequential immersion in xylene, 3 successive ethanol solutions (100, 90, and 70\%) and distilled water. As tau proteins largely have intracellular distributions, permeabilisation of nuclear and plasma membranes was carried out using detergents: Tween-20 (0.05\%: Sigma-Aldrich P1379) in citrate buffer (Citric acid, anhydrous, Sigma-Aldrich C1857-100G), and Triton-X-100 (0.1\%: Sigma-Aldrich T8787-50ML) in phosphate buffered saline (PBS: Gibco 20,012-019) forming PBST. Antigen retrieval was achieved through heating sections in a microwave (setting to $800 \mathrm{~W}$ ) in citrate buffer ( $\mathrm{pH}$ 6.0) for 5 mins before slides were left to gradually cool to room temperature (RT) for 20 mins. Following two 5 min PBS washes, sections were blocked with goat serum (Sigma-Aldrich, G9023-10 M: 1:20 in PBST) for $1 \mathrm{~h}$ at RT. After performing two $3 \mathrm{~min}$ PBST washes sections were incubated with primary anti-pTau (Thermo Scientific MN1020, mouse monoclonal AT8: 1:100 dilution in PBST) and anti-Tau (Dako, rabbit polyclonal A0024: 1:2000 dilution in PBST) antibodies for 1 $\mathrm{h}$ at RT. A0024 binds to total tau independent of phosphorylation (amino acids 243-441) whereas AT8 binds to aberrantly phosphorylated tau species $\left(\mathrm{pS}_{202} / \mathrm{pT}_{205}\right)$. Negative controls, where no primary antibody incubation was performed, were conducted alongside each experimental run (Additional file 1: Figure S2). Following three washes with PBST (5 mins each) sections were incubated with fluorophore conjugated complementary secondary antibodies at RT for $1 \mathrm{~h}$ (Alexa-Fluor 568 goat anti-mouse A21124 and Alexa-Fluor 647 goat anti-rabbit A21244, Life Technologies: 1:200 dilution in PBST). This, and all subsequent stages were conducted in the dark to prevent photobleaching. Sections underwent three further $5 \mathrm{~min}$ PBST washes, and were then incubated with Hoechst stain $(16.2 \mathrm{mM}$ stock solution diluted to 1:5000 in PBS) for 15 mins at RT to provide blue nuclear staining. Two 3 min PBS washes followed. Slides were coverslipped (Mansel-Gläser $24 \times 60 \mathrm{~mm}$ ) following the application of Vectashield anti-fade mounting medium (Vector Laboratories, H-1000) and stored at $4{ }^{\circ} \mathrm{C}$ until imaged. Fluorescent confocal microscopy (Zeiss LSM510 confocal microscope with Zen 2 software) generated qualitative and semi-quantitative data. Constant standardised settings were used for comparative non-adjusted imaging, and optimised settings used for adjusted imaging. This semi-quantitative data included the number of pTau positive cells in the RGCL and INL, and the mean intensity per pixel of areas of individual retinal strata (RGCL, IPL, INL and photoreceptor inner segment (PIS)). Data was generated by using Zen \& Image J softwares to analyse images of central and peripheral retinal regions. This data, like the semi-quantitative data acquired from H\&E images, allowed comparison between $\mathrm{rTg} 4510$ and wildtype retinas, and between the central and peripheral retina.

For immunohistochemistry in the brain, brain sections were deparaffinised and hydrated by sequential immersion in xylene, 3 successive ethanol solutions (100, 90, and 70\%) and distilled water. Antigen retrieval was performed using the Lab Vision PT module system 
(Thermo Scientific), where sections were heated to $100^{\circ}$ $\mathrm{C}$ for $20 \mathrm{~min}$ in citrate buffer (TA-250-PM1X; Thermo Scientific). Slides were transferred to a Lab Vision Autostainer (Thermo Scientific) where the following incubations were performed: 10 min in $\mathrm{H}_{2} \mathrm{O}_{2}(0.3 \%)$; 30 min in normal goat serum (1:20; Vector Laboratories); $60 \mathrm{~min}$ in primary antibody for aberrantly phosphorylated $\left(\mathrm{pS}_{409}\right)$ tau (PG-5; 1:8000 from Peter Davies, Albert Einstein College of Medicine, NY, USA); $30 \mathrm{~min}$ in biotinylated goat anti-mouse IgG (1:200, BA9200; Vector Laboratories); $30 \mathrm{~min}$ avidin-biotin complex solution (PK-7100; Vector Laboratories); 5 min in 3,3'-diaminobenzidine (SK-4105; Vector Laboratories). Apart from the last two steps, PBST was used for diluting reagents and washes between steps. Sections were then counterstained with haematoxylin before dehydration and cover-slipping. To quantify PG-5-positive tau pathology, stained sections were digitised using the Scanscope AT slide scanner (Aperio) at $20 \times$ magnification. Image $\mathrm{J}$ software was used to view the digitised tissue sections, threshold for immunoreactivity and delineate boundaries of the visual cortex, hippocampus, thalamus, superior colliculus and optic tract. Data is expressed as the percentage coverage of immunoreactivity in each region.

\section{Magnetic resonance imaging of humans Participants}

Five male subjects fulfilling the criteria for the diagnosis of behavioural variant FTD [40] and carriers of a mutation in the MAPT gene (three $10+16$ and two R406W mutations) were consecutively recruited from a tertiary referral cognitive disorders clinic at the National Hospital for Neurology and Neurosurgery, London, UK. Five healthy controls were also consecutively recruited (1 female, age range: mean(standard deviation) 62(11) years). Patients' age: 64(3) years; disease duration: 9(3) years; age at onset: 55(1) years. A full clinical history including ophthalmic history was taken from all patients as part of the standard assessment. Hence the patients (and controls) included in this study were free of any retinal or eye disease which may have confounded the data presented.

\section{Image acquisition}

Volumetric T1- and T2-weighted MRI was performed in all subjects. MRI scans were acquired on a $3 \mathrm{~T}$ scanner (Tim Trio, Siemens) with the following sequences: (i) high-resolution isotropic 3D T1-weighted MPRAGE (sagittal orientation; $\mathrm{TR}=2200 \mathrm{~ms}, \mathrm{TI}=900 \mathrm{~ms}, \mathrm{TE}=2.9$ ms, flip angle $=10^{\circ}$, acquisition matrix $=256 \times 256$ and spatial resolution $=1.1 \mathrm{~mm}$ ); and (ii) high-resolution isotropic 3D T2-weighted fast spin echo/SPACE (sagittal orientation; TR $=3200 \mathrm{~ms}$, apparent $\mathrm{TE}=105 \mathrm{~ms}$, variable refocusing pulse flip angle to achieve T2-weighting, acquisition matrix $=256 \times 256$ and spatial resolution $=$ $1.1 \mathrm{~mm})$.

\section{Image analysis}

Acquired T1-weighted images were initially transformed into standard space by a rigid registration to the Montreal Neurological Institute (MNI305) template. Acquired T2 images were registered to the MNI305 template as previously described [6]. Segmentations of the optic nerve were performed manually on about 12 consecutive coronal slices on co-registered T1- and T2MRIs using NiftyMIDAS (Centre for Medical Image Computing, UCL: http://cmic.cs.ucl.ac.uk/home/software/), starting from the most rostral slice where the eyeball was no longer visible. The intrarater intraclass correlation (ICC) for the optic nerve segmentation, computed with a two-way random effects model, was 0.85 (95\% confidence intervals: 0.50-0.96).

\section{Statistical analysis}

Statistical comparisons between animal groups were performed via either a two-way analysis of variance (ANOVA) followed by post-hoc Bonferoni post-tests for multiple comparisons, or (un)paired t-tests for single comparisons, using GraphPad Prism (v5 for Windows, San Diego, CA, USA. Statistical comparisons between patient cohorts were performed via an ANOVA followed by post-hoc Bonferoni post-tests for multiple comparisons, using GraphPad Prism (v5 for Windows, San Diego, CA, USA).). All individual data points are shown with mean \pm SEM for animals/participants in each group.

\section{Results}

pTau in the neurosensory retina of $\mathrm{rTg} 4510$ mice

Immunolabelling of total tau with the A0024 antibody in the neurosensory retina of wildtype as well as in rTg4510 mice showed cytosolic and neurite immunoreactivity that was strongest in the retinal ganglion cell layer (RGCL), inner plexiform layer (IPL) and inner nuclear layer (INL) (Fig. $1 \mathrm{a}$ and $\mathrm{d}$, respectively). Co-labelling with AT8, which labels pTau, showed occasional weak cell body labelling in wildtype animals (Fig. 1b) that co-localised with total tau (Fig. 1c and C'). The red signal observed in the photoreceptor layer on Fig. $1 \mathrm{~b}$ was the result of the combination of photoreceptor outer segment (POS) autofluorescence and nonspecific secondary antibody binding that did not differ from what was observed in control sections to which no primary antibody was added (Additional file 1: Figure S2). In contrast, in the rTg4510 animals, numerous neuronal somas and neurites were pTau immunopositive in the RGCL, IPL and INL (Fig. 1e) that showed co-localisation throughout, with more intense total tau labelling (Fig. 1f, 

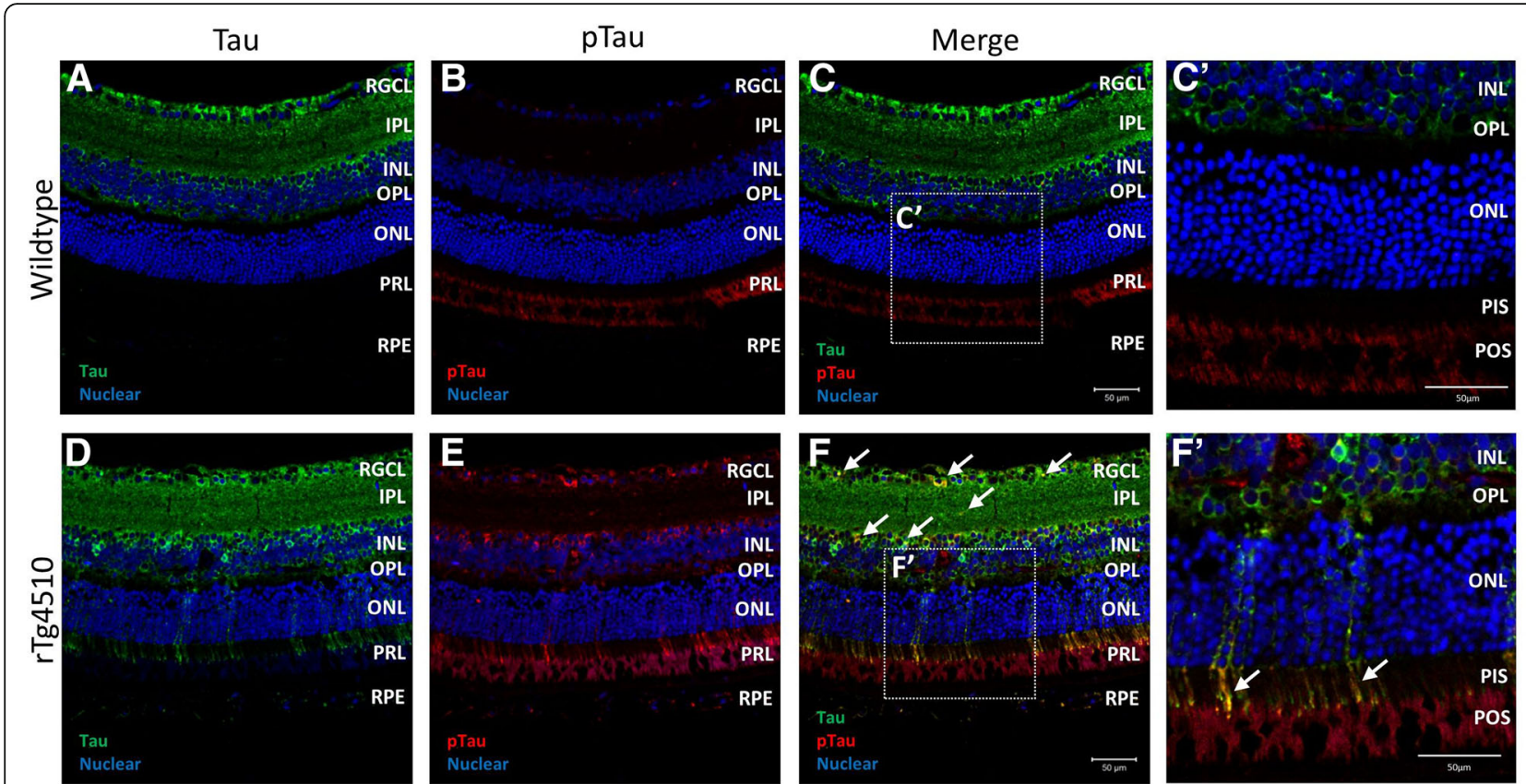

Fig. 1 Tau and pTau Immunoreactivity within the Neurosensory Retina of rTg4510 Mice. Representative immunofluorescence images of tau and pTau in the neurosensory retina of wildtype (a-c) and $\mathbf{r g} 4510$ (d-f) mice. Tau immunoreactivity is shown in green, pTau immunoreactivity is shown in red, and Hoechst nuclear counterstain is shown in blue. Co-localisation of tau and pTau stains indicated with white arrow in merged channel images (c and $\mathbf{f}$ ), which can be observed more clearly in higher magnification images ( $C^{\prime}$ and $F^{\prime}$ ). Scale bars equal to $50 \mu \mathrm{m}$

white arrows) and irregular clusters extending to the PIS were also observed (Fig. 1F,' white arrows).

Quantification of the number of cell bodies positive for pTau as a percent of total cell nuclei numbers that were counted as Hoechst positive labelling, showed a significantly higher proportion of pTau immunopositive cells in the RGCL of the central $(p<0.05)$ and peripheral $(p<0.001)$ retinas of $\mathrm{rTg} 4510$ compared to wildtype mice (Fig. 2a). Upon examination of several serial confocal images (z-stacks), pTau and tau inclusions in the RGCL and INL were shown to have morphologies reminiscent of flame-shaped inclusions of tangle-like tau (Fig. 2b, white arrows). When comparing overall average pixel intensities in pTau immunofluorescence in the central retinas between wildtype and $\mathrm{rTg} 4510$ mice, we found significantly higher labelling in the PIS $(p<0.01)$, but no significant difference in RGCL, IPL and INL in the transgenic animals (Fig. 2c). However, when the peripheral retina was compared, the RGCL, IPL INL, and the PIS, showed significantly greater pTau burden in the rTg4510 compared to the wildtype mice $(\mathrm{p}<0.01, \mathrm{p}<$ $0.01, p<0.05$ and $p<0.001$ respectively) (Fig. $2 \mathrm{~d}$ ). When comparing pTau immunofluorescence intensities between the central and peripheral retina of the $\mathrm{rTg} 4510$ mice, there was a trend towards higher intensities in peripheral retina of all layers but only the IPL and INL showed statistically significant higher pTau staining intensities (both $\mathrm{p}<0.05$ ) (Fig. 2e). Lastly, in $\mathrm{rTg} 4510$ retinas stained with pTau and total tau, labelling was consistently seen in axons of the RNFL (Fig. 1f), which ultimately amass to form the optic nerve.

\section{Decrease in RGC densities in rTg4510 mice}

The nuclear density of the RGCL was evaluated on H\&E stained $4 \mu \mathrm{m}$ paraffin embedded sections. RGC numbers were significantly reduced in the central but not in the peripheral retina of $\mathrm{rTg} 4510$ compared to wildtype animals $(p<0.001)$ (Fig. 3a). The RGC numbers were lower in the periphery compared to the central retina in both wildtype and $\mathrm{rTg} 4510$ animals $(\mathrm{p}<0.001)$ (Fig. 3a). When comparing the thickness of the IPL, no differences between the central and peripheral retina, nor between animal groups was observed (Fig. 3b). No differences were observed in the nuclear density of the INL between wildtype and $\mathrm{rTg} 4510$ mice, although there were significantly fewer cells present in the retinal periphery in all mice (Fig. 3c).

\section{Neurodegeneration in the visual system of $\mathrm{rTg} 4510$ mice} To examine whether there were tau induced neurodegenerative changes in parts of the visual system, brain T2-weighted MR images were examined (Fig. 4a). Segmentation of the optic nerve demonstrated a significant reduction in normalised (to total eye volume) optic nerve volume in $\mathrm{rTg} 4510$ mice compared to wildtype animals $(p<0.05)$ (Fig. 4b) (individual non-normalised 


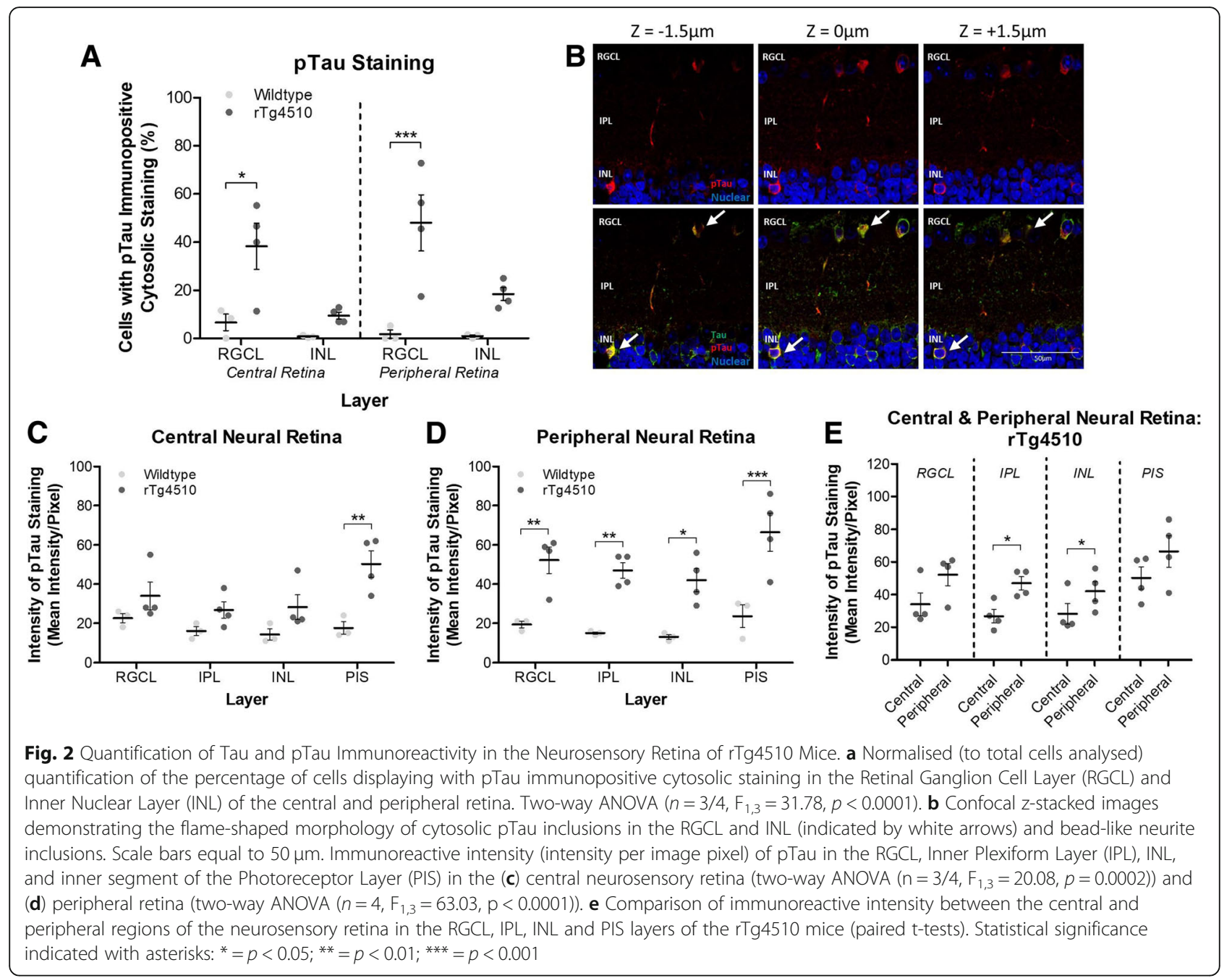
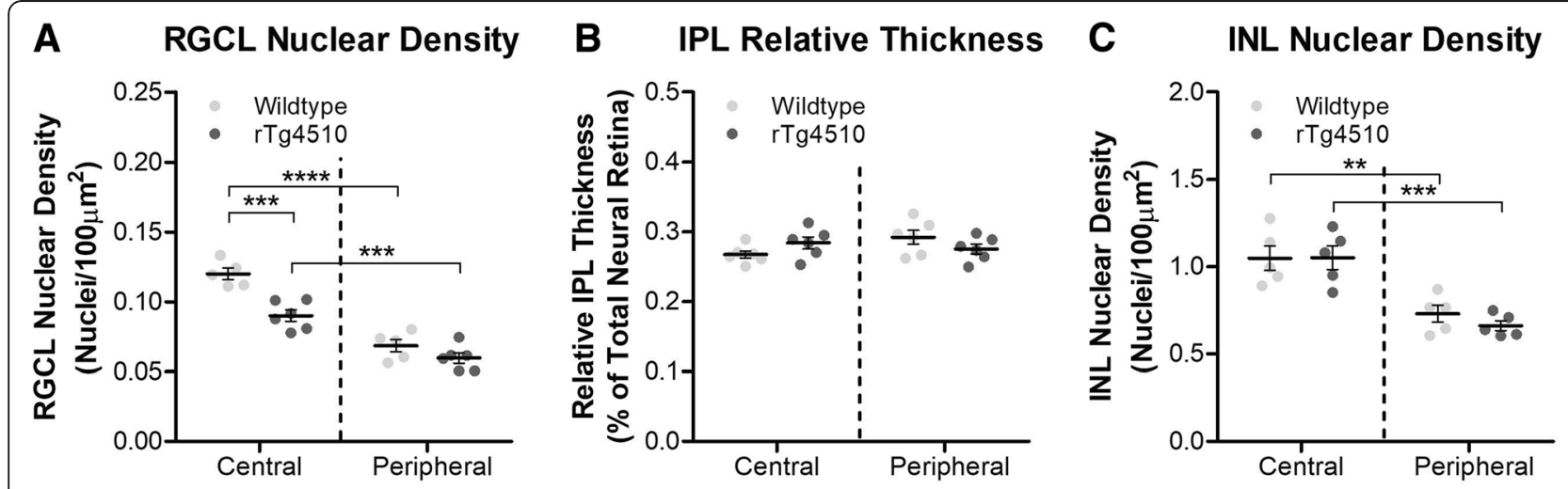

Fig. 3 - Neurodegeneration Within Layers of the Neurosensory Retina of rTg4510 Mice. a Differences in nuclear density (nuclei per tissue area) in the Retinal Ganglion Cell Layer (RGCL) of the central and peripheral retina of wildtype and rTg4510 mice. Two-way ANOVA $\left(n=6, F_{1,1}=22.73, p=\right.$ 0.0002). b Relative thickness (\% of total neural retina) of the Inner Plexiform Layer (IPL) of the central and peripheral retina of wildtype and rTg4510 mice, as ascertained from H\&E staining. Two-way ANOVA ( $\left.n=6, F_{1,1}=0.00002853, p=0.9958\right)$. c Differences in nuclear density (nuclei per tissue area) in the Inner Nuclear Layer (INL) of the central and peripheral retina of wildtype and rTg4510 mice. Two-way ANOVA $\left(n=6, F_{1,1}=\right.$ $0.3358, p=0.5704)$. Statistical significance indicated with asterisks: ${ }^{* *}=p<0.01{ }^{* * *}=p<0.001,{ }^{* * *}=p<0.0001$ 

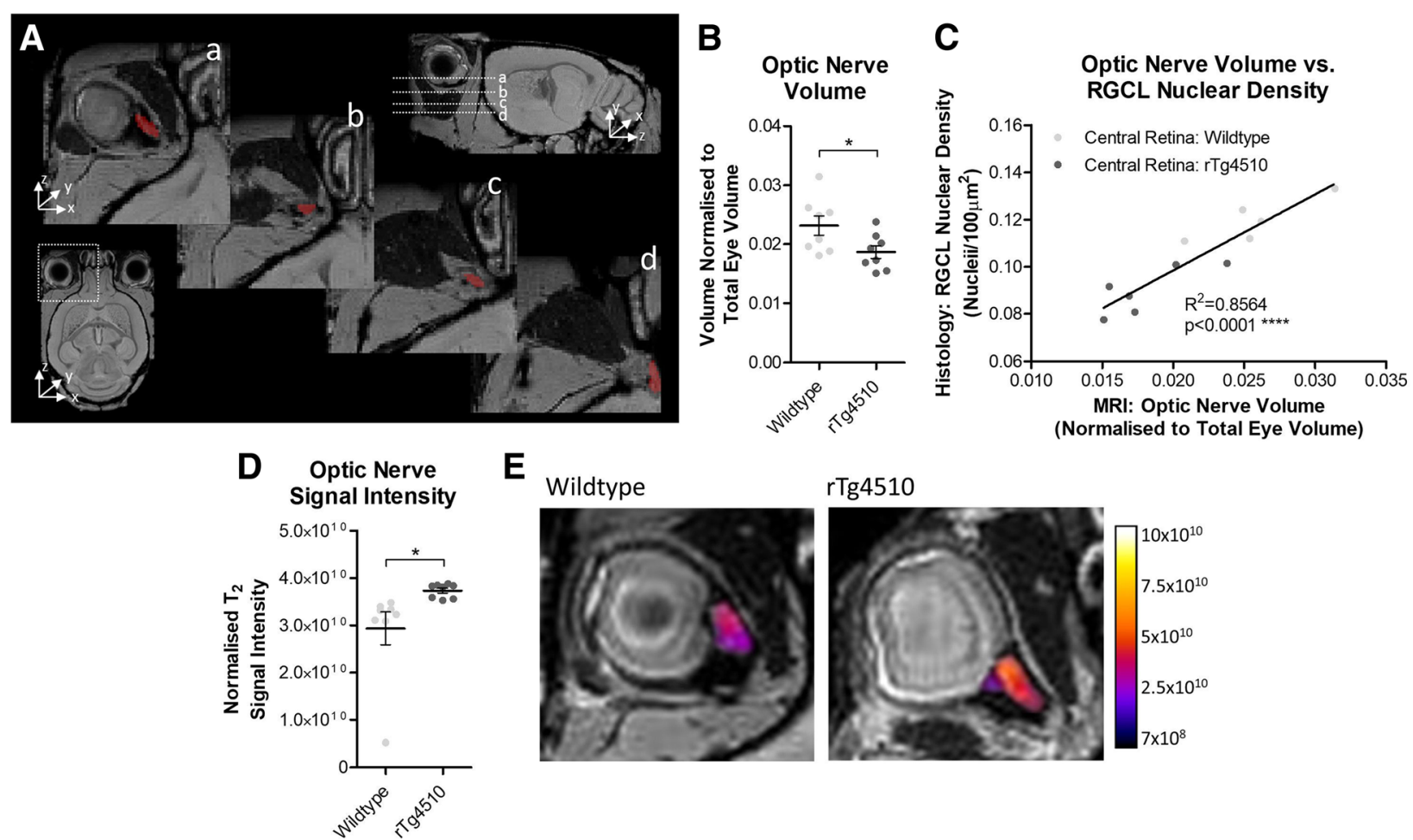

E Wildtype

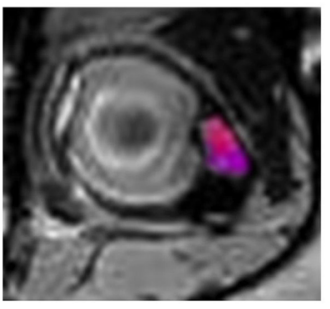

rTg4510

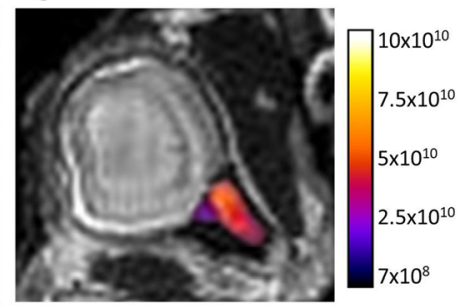

Fig. 4 Reduced Optic Nerve Volume in rTg4510 Mice Ascertained Through MRI. A Example images demonstrating the segmentation protocol used for volumetric analysis of the optic nerve from T2-weighted MR images. (a, b, c and d) Different horizontal levels of the 3D MR images, with the optic nerve highlighted in red. B Normalised (to total eye volume) volume of the optic nerve in wildtype and rTg4510 mice. Unpaired t-test ( $n=8, p=0.0375)$. Un-normalised and total eye volumes are shown in Additional file 1: Figure S4. C Correlation between MRI derived measures of optic nerve volume, and RGCL nuclear density derived from histology. Linear regression analysis $\left(F_{1,9}=53.66, p<0.0001\right)$. (D) Difference in normalised T2 signal in the optic nerve of wildtype and rTg4510 mice. Unpaired t-test ( $n=8, p=0.0393$ ). (E) Representative T2-weighted MR images of a cross-section of the optic nerve, with the optic nerve region pseudo colour scaled for signal intensity. Statistical significance indicated with asterisks: ${ }^{*}=p<0.05,{ }^{* * *}=p<0.0001$

optic nerve and total eye volumes are shown in Additional file 1: Figure S4). We also showed that optic nerve volume was closely correlated with RGCL nuclear density $\left(R^{2}=0.8564, p<0.0001\right)$ (Fig. 4c). In parallel, the optic nerve of rTg4510 mice presented with a greater signal intensity on T2-weighted MR images $(p<0.05)$ (Fig. 4d and e), suggestive of increased water content or oedema within the optic nerve of rTg4510 animals, which is consistent with the hypothesis of axonal degeneration.

Parcellation of brain regions involved in visual processing and memory from T2-weighted MR images of the mouse brain (Fig. 5a) revealed significant atrophy within the cortex (encompassing the visual cortex) $(p<$ $0.001)$ and the hippocampus $(p<0.001)$ of rTg4510 mice compared to wildtype, but not in the thalamus (encompassing the lateral geniculate nucleus) or superior colliculus (Fig. 5b). Furthermore, when the volumes of these regions were normalised to total brain volume, significant atrophy was still apparent in the cortex $(p<$ $0.001)$ and hippocampus $(p<0.01)$ indicative of atrophy over-and-above that experienced by the whole brain.
The thalamus (encompassing the lateral geniculate nucleus) of the rTg4510 mice however exhibited a significantly larger normalised volume than the wildtype mice $(\mathrm{p}<0.01)$, indicative of sparing of this brain region in the rTg4510 mouse in relation to the extent of whole brain atrophy (Fig. 5d). A similar, albeit not significant difference was also observed in the normalised volume of the rTg4510 superior colliculus (Fig. 5d). These observed reductions in volume within the cortex and hippocampus, and sparing of the thalamus in this model were consistent with the localised expression of mutant MAPT gene and deposition of tau pathology in the brain (Fig. 5e and f), indicative of tau induced neurodegeneration. More specifically, significantly greater levels of pTau immunoreactivity were observed in the visual cortex $(p<0.001)$, and hippocampus $(\mathrm{p}<0.001)$ of rTg4510 mice compared to wildtype animals (Fig. $5 \mathrm{~g}$ ). Subtle increases in the level of pTau staining in the rTg4510 thalamus (encompassing the lateral geniculate nucleus), superior colliculus, and optic tract, were also observed, however these differences were not significant. Tau induced neurodegeneration within the brain of these animals is also 

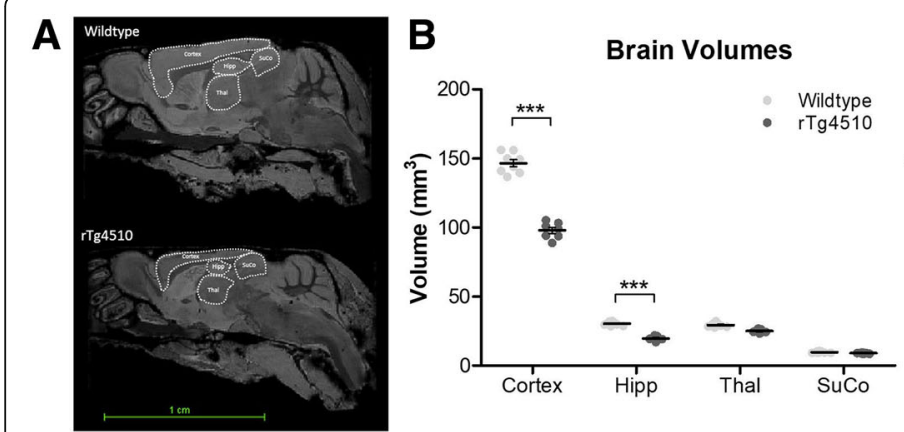

E Wildtype-PG-5-pTau

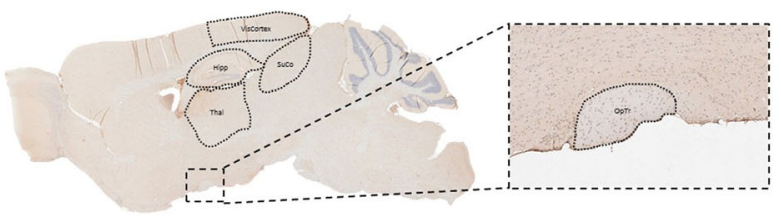

F $\quad r \operatorname{Tg} 4510-P G-5-p T a u$

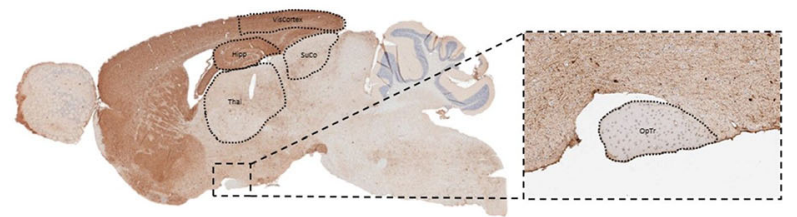

C

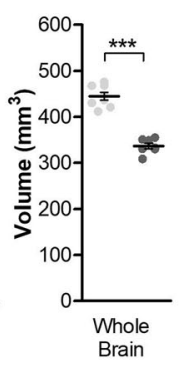

G

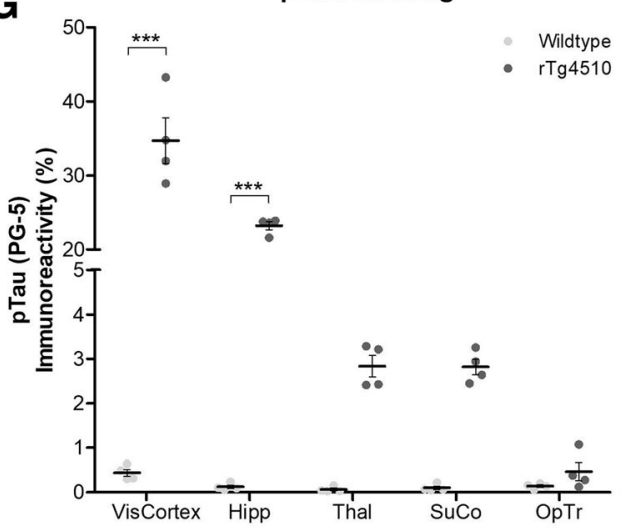

Fig. 5 - Tau Deposition and Neurodegeneration of Visual Pathways in the Brains of rTg4510 Mice. a Representative MR images of the brains of wildtype and rTg4510 mice displayed in the sagittal plane, with the visual cortex, hippocampus, thalamus and superior colliculus delineated. $\mathbf{b}$ Volumes of the cortex, hippocampus (Hipp), thalamus (Thal) and superior colliculus (SuCo) as ascertained through automated parcellation of MR images. Two-way ANOVA $\left(n=8, F_{1,3}=307.7, p<0.0001\right)$. c Wildtype and $r T g 4510$ animal whole brain volumes extracted from MR images.

Unpaired t-test $(n=8, p<0.0001)$. d Regional volumes displayed in (b) normalised to whole brain volume displayed in (c). Two-way ANOVA ( $n=$ $8, F_{1,3}=45.30, p<0.0001$ ). Representative PG-5 immunoreactivity in the (e) wildtype and (f) rTg4510 brain, with visual cortex (VisCortex), hippocampus (Hipp), thalamus (Thal), superior colliculus (SuCo) and optic tract (OpTr) delineated demonstrating forebrain localisation of pTau, and for extraction of immunoreactivity data presented in (g) pathology in the brain. Two-way ANOVA $\left(n=8, F_{1,4}=404.1, p<0.0001\right)$. Statistical significance indicated with asterisks: ${ }^{* *}=p<0.01 ;{ }^{* *}=p<0.001$

consistent with the significant amounts of both total and phosphorylated tau species detected in CSF extracts from rTg4510 mice, but not in wildtype animals (Additional file 1: Figure S5).

\section{Neurodegeneration in the optic nerve of frontotemporal dementia patients}

Given the specific atrophy observed in the optic nerve, and its relationship with RGCL nuclear density in the rTg4510 mouse model (Fig. 4c), we sought to determine whether such changes also occur in FTD patients. The volumes of the left and right optic nerves were manually segmented on co-registered T1- and T2-weighted brain MR images of MAPT mutation carrying FTD patients, and age matched controls (Fig. 6a). Segmentations revealed a significantly smaller volume of the optic nerve in FTD patients compared to age matched controls $(p<$ 0.01, in both left and right) (Fig. 6b). Moreover, these significant differences persisted after optic nerve volumes were normalised to total intracranial volume
(Additional file 1: Figure S6), indicative of atrophy over-and-above that experienced by the whole brain in these patients $(p<0.01$, in both left and right) (Additional file 1: Figure S6B).

\section{Discussion}

The overall finding of this study was that the visual disturbances in FTD sufferers might be, at least partly, due to tau induced degeneration in the neurosensory retina and optic nerve. We demonstrated this through detailed study of the eye and optic nerve of the rTg4510 mouse model of FTD and compared observed changes to those in the brain of the same animal. We also found that changes observed in the mouse optic nerve were similarly observed in human FTD patients, suggesting that the optic nerve and the neurosensory retina may be prone to tauopathic changes in FTD, and inclusion of retinal and optic nerve examination in FTD should be considered in the future. 


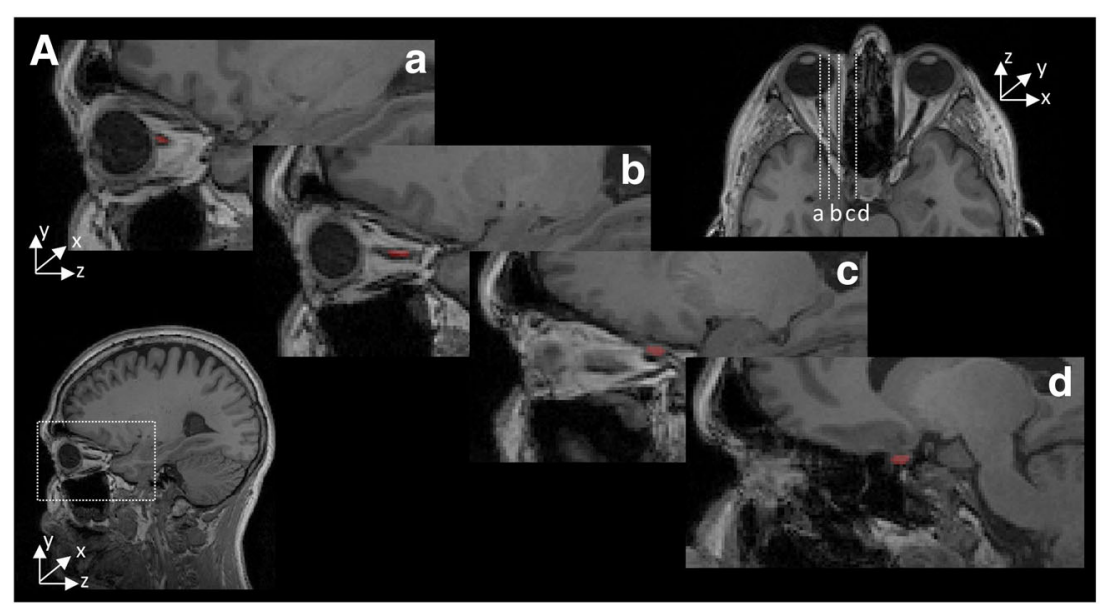

\section{B Optic Nerve Volume}

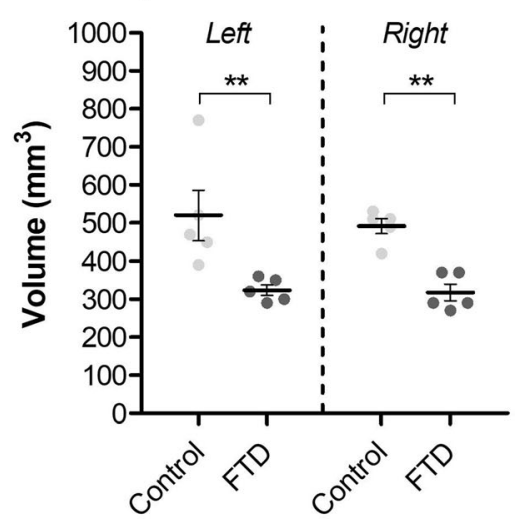

Fig. 6 - Reduced Optic Nerve Volume in MAPT Mutation Carrying FTD Patients. A Example images demonstrating the segmentation protocol used for volumetric analysis of the optic nerve from T1-weighted MR images. (a, b, c and d) Different sagittal levels of the 3D MR images, with the optic nerve highlighted in red. B Volume of the left and right optic nerves of FTD patients and age-matched healthy controls. Two-way ANOVA $\left(n=5, F_{1,1}=25.57, p=0.0001\right)$. Statistical significance indicated with asterisks: ${ }^{* *}=p<0.01$

In the rTg4510 mouse eye, hyperphosphorylated tau pathology was observed mainly in the RGCL, IPL, and INL, with additional irregular clusters within the inner segment of the photo receptor layer (PRL), translating to a significant reduction of nuclear density in the RGCL. This phenomenon was observed to be more pronounced in the peripheral as opposed to the central retina in most layers. The significance of such anatomical organisation of tauopathy in the retina is yet to be fully understood, however this regional nature of retinal pathology observed here in the $\mathrm{r} \operatorname{Tg} 4510$ eye mirrors the pattern of pathology we observed recently in the retina in AD [11]. The axons of RGCs in this layer converge in the RNFL to form the optic nerve, where we observed an elevated T2 signal, indicative of oedema/demyelination, and a decrease in volume. Unsurprisingly, this optic nerve atrophy was associated with RGCL nuclear density loss. Most interesting however, was that similar optic nerve atrophy was observed in cases of human FTD, suggesting that the optic nerve, and by extension, the RGCL, are prone to tauopathic changes in FTD. The consequence and pathophysiological relevance of such changes depend upon the context of cell populations affected by tauopathy. While the RGCL is composed largely of RGCs, displaced amacrine cells (ACs) are suggested to encompass up to $59 \%$ of the total cells in this layer [38]. ACs are distinguishable from RGCs by their smaller soma and lack of projecting axons in the RNFL, hence these cells are unlikely to contribute directly to the optic nerve atrophy observed here. Hence rather, cells affected by the increase in pTau immunoreactivity and decrease in nuclear density in the RGCL are likely to be RGCs in origin. That being said, double staining for pTau and markers of RGCs themselves in this retinal layer in the $\mathrm{rTg} 4510$ is required in order to confirm such a hypothesis. The INL-IPL border on the other hand is itself dominated by ACs with around 1.7\% of the cell nuclei here belonging to displaced RGCs [37]. Whether the pTau immunopositive cells identified at the INL-IPL border are actually ACs or displaced RGCs needs further investigation. Yet it would appear, at least from data presented here from the rTg4510 mouse model of tauopathy, that RGC and AC cell populations in the neurosensory retina are the most intrinsically susceptible to tau mediated pathology.

The morphology of RGC and AC pTau inclusions observed in our study compare favourably to NFT pathology found in the brains of other mouse models of FTD and human FTD itself. For example, Deters at al. [12], describe the same AT8 positive flame-shaped and bead like structures of pTau observed in our study (Fig. 2b) as NFTs and somatodendendritic tau inclusions, in the mThy1.2.hTau.P301L mouse brain. Similar morphological expression of pTau has also been described in brains of FTD patients carrying the same P301L mutation in the MAPT gene [45]. The prevailing hypothesis from the literature is that such cytoplasmic aggregations of pTau are pathophysiological in nature [4], and hence we hypothesise that the pTau pathology observed in the RGCL in the rTg4510 mouse would be detrimental to the neuronal physiology of resident RGCs. Indeed this idea would be consistent with previous findings from Mazzaro et al., [27], in which reduced activity, assessed through the use of a pattern electroretinogram (pERG) was observed in tau laden RGCs in 5 month old mThy1.2.hTau.P301S mice. Further to this we have observed a significant reduction in nuclear density in this cell layer of the rTg4510 retina, and hence this would 
suggest that the pTau labelling observed in the RGCL in the rTg4510 mouse is capable of inducing neurodegeneration within this region.

From the retina, axons of RGCs project, via the RNFL and optic nerve to the brain. Given the reduced volume of the optic nerve in $\mathrm{rTg} 4510$ mice, and the association of this measure with RGCL nuclear density, this data would suggest tau induced neurodegeneration of RGCs in the retina: with both somal and axonal reductions. In the current study we have not addressed as to whether or not the optic nerve itself is burdened by tau pathology, yet we show that the optic nerve, along with being smaller in rTg4510 mice, is associated with a greater $\mathrm{T} 2$ signal. T2 signal is influenced by proton transfers, molecular exchange and diffusion of water, and hence an elevated T2 signal is suggestive of increased water content or oedema within the optic nerve of $\operatorname{rTg} 4510$ animals, which is consistent with the hypothesis of neurodegeneration within this region. Moreover, due to the nature of the axonal projections from the RGCs within the eye to the brain, the optic nerve contains a dense packed myelin sheath around the axonal fibres [35]. The hydrophobic properties of the lipidic bilayer in myelin restricts molecular motion of protons [5, 29] and hence hypointensity on T2-weighted MR images reflects larger myelin content. The hyperintensity observed on T2-weighted MR images here then would therefore also be consistent with demyelination of the optic nerve in the $\mathrm{r} \operatorname{Tg} 4510$, a phenomenon which has been previously described in white matter regions of the $\mathrm{rTg} 4510$ mouse brain [33, 42]. These quantitative MR findings add weight to the suggestion that indeed neurodegeneration of the RGC axons in the optic nerve is taking place in the $\operatorname{rg} 4510$ mouse.

MAPT P301L expression in the $\mathrm{rTg} 4510$ mouse is CaMKIIa promoter driven and hence it may well be possible that pTau localisation is due to CaMKIIa expression in RGCs and AC populations. That being said, the CaMKIIa expression profile in the mouse neurosensory retina has not been fully elucidated in the literature. In the rat retina, it has been shown that conventionally placed and displaced ACs are CaMKIIa positive [34], and in the primate retina, CaMKIIa expressing RGCs have been shown to exist [7]. It may well be that the specific promoter system employed in this transgenic mouse causes expression of pTau within these cell layers. Indeed, further assessment of tau pathology in the rTg4510 eye should aim to determine whether or not this is the case, through regional and cell specific profiling of CaMKIIa expression in the mouse retina, to understand the contribution of promoter-driven transgene expression in the deposition of pTau pathology in affected population in the mouse neural retina. Curious however, that similar patterns of expression were also noted in both the mThy1.2 and PRNP promoter driven P301 mutant mouse lines $[14,18]$, suggesting the specific vulnerability of these retinal cell layers, rather than promoter driven expression. Likewise, photoreceptors are unlikely to express CaMKIIa, and yet tau and pTau were both observed in this layer in the $\mathrm{r} \operatorname{Tg} 4510$ mouse retina. This raises the intriguing possibility therefore that mechanisms of tau deposition other than promoter-driven expression, may similarly be occurring in the $\mathrm{rTg} 4510$ mouse retina.

It is known that tau propagates in the brain between functionally connected regions [1]. More specific to the visual pathways, it was noted recently by Mazzaro et al. [27], that after tau containing diseased brain homogenate was injected into the Superior Colliculus of mThy1.2.hTau.P301S mice, augmented cerebral, as well as optic nerve pathological tau was evident. The retinal expression pattern of CaMKIIa and promoter-driven tau expression notwithstanding, these data suggest that the retinal tau pathology observed here in the rTg4510 mouse could indeed be a consequence of tau propagation, via the optic nerve, to the retina itself, from functionally connected regions of the visual pathways. As previously mentioned, the expression of the human $M A P T$ gene harbouring the P301L mutation in this animal model is driven by the CaMKIIa promoter system (Fig. 7a) and hence tau pathology is observed largely within areas where CaMKIIa is expressed most highly, i.e. the forebrain of mice (Fig. $5 \mathrm{f}$ and g), resulting in proportional atrophic changes in brain volume (Fig. 5a-d), as previously observed in this model [47]. Appropriately, we show here that the cortex, including the visual cortex, of the 7.5 month old rTg4510 mouse is heavily burdened by tau pathology (Fig. 5). Yet the subcortical areas involved in visual processing, i.e. the super colliculus and lateral geniculate nucleus, are not overtly affected by either tau pathology and hence nor neurodegeneration, but tau levels appear to be increased compared to controls (Fig. 5g). It is possible therefore, that the tau pathology observed in the retina is a result of propagation from the visual cortex via the geniculate and extra-geniculate visual pathways in the brain (Fig. 7c). This would be consistent with findings of the propagation of tau in the brain from groups such as Ahmed at al. [1], and Mazzaro et al. [27]. Moreover, the incidental finding of the presence of pTau in the PRL, where promoter expression is lacking, would too support, however not prove, the idea that pTau driven pathology in the neurosensory retina is a result of propagation.

In the current study we observed reduced optic nerve volume in FTD patients. Similarly, in a mouse model of the disorder, we demonstrated that reduced optic nerve volume is accompanied by likely optic nerve demyelination (T2 hyperintensity), and associated with a 


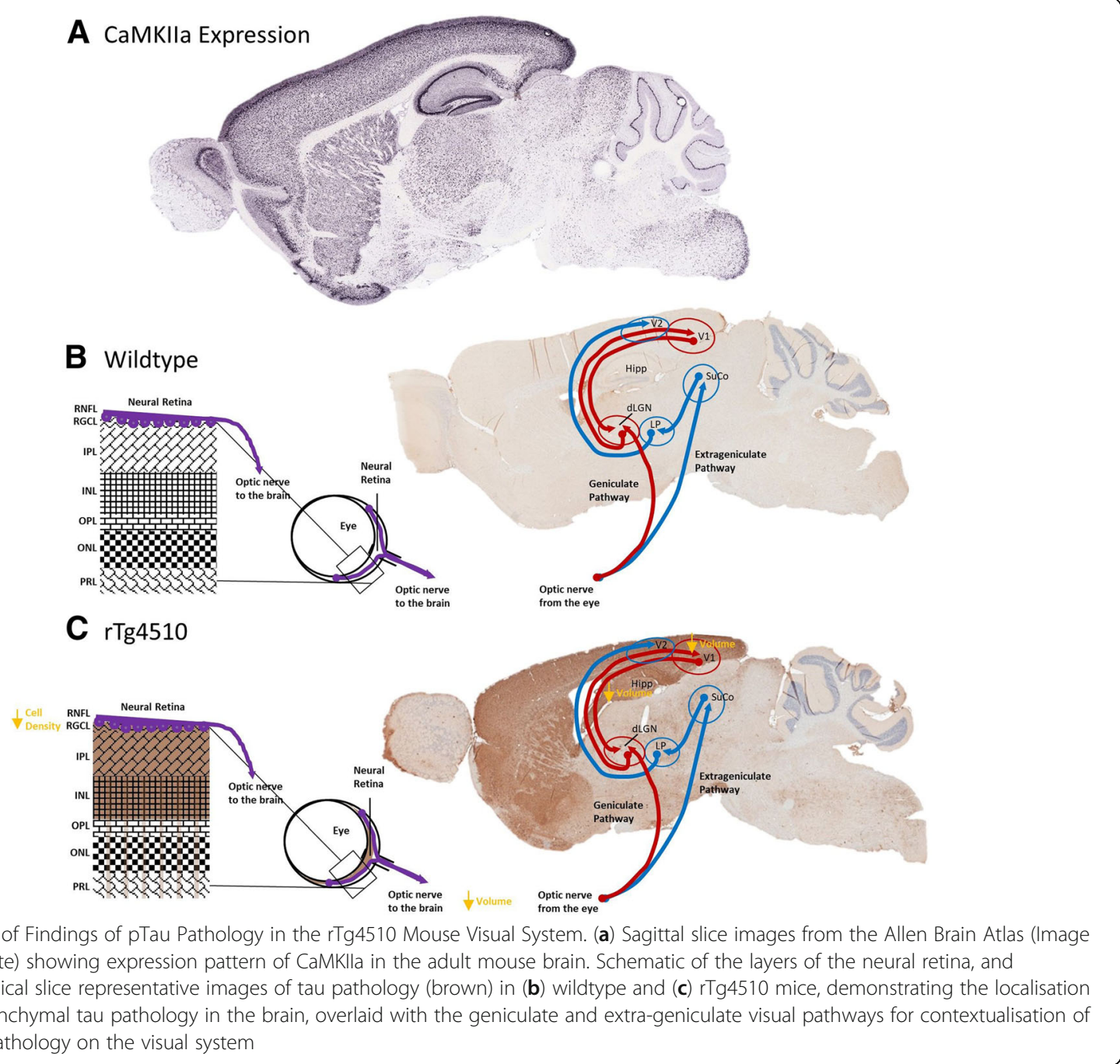

reduction in nuclear density of the tau burdened RGCL. Whether similar levels of neurodegeneration and reduction of RGCL nuclear density take place in the eyes with FTD sufferers will need to be examined in clinical studies, however our finding of reduced optic nerve volume and changes within the neural retina in the rTg4510 mouse model, would recommend assessment of retinal layer thicknesses in FTD. The literature regarding retinal layer thicknesses in FTD patients however is limited and mixed. Specifically, using optic coherence tomography (OCT), Ferrari et al. [13] demonstrate, consistent with the pattern of tau pathology we observed in the mouse eye, NFL and RGCL-IPL layer thinning in FTD compared to healthy controls. Whereas Kim et al. [23], report that there is no difference between inner retinal layer thicknesses of FTD patients compared to control, but that the retinal outer layer is thinner in FTD patients. Interestingly however, a study by Albrecht et al. [2], reveals that patients with another degeneratiuve taupathy, progressive supranuclear palsy, exhibit reduced RGCL, IPL, and outer nuclear layer thicknesses, but greater thickness in the outer plexiform layer compared to control. All the aforementioned studies however only report changes in layer thicknesses rather than any quantitication or localisation of retinal tau pathology. If it is the case though, as supported by the mouse data reported here, that in clinical FTD tau accumulates in layers such as the RGCL leading to RGC neurodegeneration, findings from the glaucoma field would suggest that altered vision is a likely consequence [3]. Together with the atrophic changes taking place in the visual cortex, our findings that the retina and the optic nerve are directly affected by tau pathology may explain the wealth of data linking cognitive visual changes to tauopathies $[9,10,15$, $21,28]$ and may suggest that more attention should be paid towards retinal and optic nerve changes in FTD in the clinic. Eye imaging is significantly simpler, faster, and better tolerated than brain imaging. Retinal imaging may therefore serve as a surrogate marker for disease progression, benefiting both patients and the health system enormously. 
In conclusion, concurrent degeneration within the neurosensory retina and reduced volume within the optic nerve of rTg4510 mice suggests that tau induced degeneration may play a role in affecting signal propagation to the brain in this animal model. The volume loss of the optic nerve in FTD patients suggests that neurosensory retinal tau pathology may too exist in the eyes of FTD patients, however this would need to be evaluated and confirmed in cadaveric eyes from FTD patients, as well as clinical OCT imaging in FTD sufferers. Currently, the consequences of such pathologies is not known, but preventing and protecting tauopathy patients from the cognitive visual changes routinely experienced with disease development may represent a novel approach for improving quality of life of FTD sufferers, and decrease the burden on carers and society by preserving independent living for longer. Furthermore, it is intriguing to speculate whether optic nerve degeneration could be an early pathological sign of FTD, which could be examined in further longitudinal studies, and which would recommend inclusion of retinal and optic nerve examination in FTD.

\section{Additional file}

Additional file 1: Figure S1. Definition of the Central and Peripheral Retina. Figure S2. Red Channel Autofluorescence of the Outer Segment of the Photoreceptor Layer. Figure S3. Non-normalised Quantification of pTau Immunoreactivity in the Neurosensory Retina of rTg4510 Mice. Figure S4. Non-normalised Optic Nerve and Total Eye Volumes of rTg4510 and Wildtype Mice. Figure S5. Tau Cerebrospinal Fluid Biomarker in rTg4510 Mice. Figure S6. Optic Nerve Volume of FTD Patients Normalised to Total Intracranial Volume. (DOCX $570 \mathrm{~kb}$ )

\section{Acknowledgements}

Not applicable.

\section{Funding}

The Dementia Research Centre is supported by Alzheimer's Research UK, Brain Research Trust, and The Wolfson Foundation. This work was supported by the NIHR Queen Square Dementia Biomedical Research Unit and the NIHR UCL/H Biomedical Research Centre, the MRC UK GENFI grant and the Alzheimer's Society.

IFH is funded by a research grant from Eli Lilly and Company, and the Engineering and Physical Sciences Research Council (EPSRC) UK (EP/ N034864/1).

JMO'C is funded by UK Medical Research Council (MRC) Doctoral Training Grant Studentship (MR/J500422/1).

LC is funded by an unrestricted grant from OPTOS Plc. Ltd.

DM is funded by a EPSRC studentship from UCL's Centre for Doctoral Training in Medical Imaging.

JDR is supported by an MRC Clinician Scientist Fellowship (MR/M008525/1) and has received funding from the NIHR Rare Disease Translational Research Collaboration (BRC149/NS/MH).

MFL receives funding from BBSRC/AstraZeneca Industrial Partnership Studentship (BB/E528979/1), the UK Regenerative Medicine Platform Safety Hub (MR/K026739/1), Eli Lilly and Company, and the EPSRC (EP/N034864/1). IL receives funding from the Bill Brown Charitable Trust, Moorfields Eye Hospital Special Trustees and Mercer Fund from Fight for Sight.

\section{Availability of data and materials}

The datasets used and/or analysed during the current study available from the corresponding author on reasonable request.

\section{Authors' contributions}

IFH analysed animal MR images, compiled the figures and wrote the manuscript. RW, PMB and LC performed immunohistochemical analysis of mouse retinas. JMO'C acquired the animal MR images, and DM performed mouse brain parcellation. MB and JDR segmented and analysed human MR images. AF and ZA performed immunohistochemical analysis of mouse brains. TKM and MJO'N provided the mice for the study. MFL and IL conceived the project, designed the experiments, led the analysis for the study and contributed to the writing of the manuscript. All authors read and approved the final manuscript.

\section{Ethics approval and consent to participate}

For human data, the study was approved by the local ethics committee (Queen Square Neurology Research Ethics Committee) and written informed consent was obtained from all participants. For animal data, all the experiments were performed in accordance with the Animals (Scientific Procedures) Act 1986 (ASPA) revised according to the European Directive 2010/63/EU and the UK Home Office (Scientific Procedures) Act (1986) with prior project approval from UCL's internal Animal Welfare and Ethical Review Body.

Consent for publication

Not applicable.

\section{Competing interests}

AF, ZA, TKM and MJO'N are all/were full time employees of Eli Lilly and Company.

\section{Publisher's Note}

Springer Nature remains neutral with regard to jurisdictional claims in published maps and institutional affiliations.

\section{Author details \\ ${ }^{1}$ UCL Centre for Advanced Biomedical Imaging, Division of Medicine, University College London, Paul O'Gorman Building, 72 Huntley Street, London WC1E 6DD, UK. ${ }^{2}$ UCL Institute of Ophthalmology, University College London, 11-43 Bath Street, London ECIV 9EL, UK. ${ }^{3}$ Dementia Research Centre, UCL Institute of Neurology, University College London, National Hospital for Neurology and Neurosurgery, London WC1N 3BG, UK. ${ }^{4}$ Centre for Experimental Medicine, The Queen's University Belfast, Belfast BT9 7BL, UK. ${ }^{5}$ School of Engineering Science, Simon Fraser University, 8888 University Drive, Burnaby, BC V5A 156, Canada. ${ }^{6}$ Eli Lilly and Company, Erl Wood Manor, Windlesham, Surrey GU20 6PH, UK.}

Received: 13 December 2018 Accepted: 20 December 2018 Published online: 07 January 2019

\section{References}

1. Ahmed Z et al (2014) A novel in vivo model of tau propagation with rapid and progressive neurofibrillary tangle pathology: the pattern of spread is determined by connectivity, not proximity. Acta Neuropathol 127(5):667683

2. Albrecht P et al (2012) Optical coherence tomography in parkinsonian syndromes. PLoS One 7(4):e34891

3. Almasieh $\mathrm{M}$ et al (2012) The molecular basis of retinal ganglion cell death in glaucoma. Prog Retin Eye Res 31(2):152-181

4. Avila J et al (2006) Tau phosphorylation, aggregation, and cell toxicity. J Biomed Biotechnol 2006:74539

5. Barkovich AJ (2000) Concepts of myelin and myelination in neuroradiology. Am J Neuroradiol 21(6):1099

6. Bocchetta $\mathrm{M}$ et al (2015) Detailed volumetric analysis of the hypothalamus in behavioral variant frontotemporal dementia. J Neurol 262(12):2635-2642

7. Calkins DJ, Sappington RM, Hendry SHC (2005) Morphological identification of ganglion cells expressing the a subunit of type II calmodulin-dependent protein kinase in the macaque retina. J Comp Neurol 481(2):194-209

8. Chiasseu $\mathrm{M}$ et al (2016) Tau accumulation, altered phosphorylation, and Missorting promote neurodegeneration in Glaucoma. J Neurosci 36(21):5785 
9. Cronin-Golomb A (1995) Vision in Alzheimer's disease. The Gerontologist 35(3):370-376

10. Cronin-Golomb A et al (1991) Visual dysfunction in Alzheimer's disease: relation to normal aging. Ann Neurol 29(1):41-52

11. Csincsik $L$ et al (2018) Peripheral retinal imaging biomarkers for Alzheimer's disease: a pilot study. Ophthalmic Res 59(4):182-192

12. Deters N, Ittner LM, Götz J (2008) Divergent phosphorylation pattern of tau in P301L tau transgenic mice. Eur J Neurosci 28(1):137-147

13. Ferrari $L$ et al (2017) Optical coherence tomography reveals retinal Neuroaxonal thinning in frontotemporal dementia as in Alzheimer's disease. J Alzheimers Dis 56(3):1101-1107

14. Gasparini L et al (2011) Tau inclusions in retinal ganglion cells of human P301S tau transgenic mice: effects on axonal viability. Neurobiol Aging 32(3):419-433

15. Gilmore GC, Whitehouse PJ (1995) Contrast sensitivity in Alzheimer's disease: a 1-year longitudinal analysis. Optom Vis Sci 72(2):83-91

16. Goedert M, Crowther RA, Spillantini MG (1998) Tau Mutations Cause Frontotemporal Dementias. Neuron 21(5):955-958

17. Gupta N et al (2008) Retinal tau pathology in human glaucomas. Can J Ophthalmol 43(1):53-60

18. Ho WL et al (2015) Investigating degeneration of the retina in young and aged tau P301L mice. Life Sci 124:16-23

19. Ho W-L et al (2012) Review: Tauopathy in the retina and optic nerve: does it shadow pathological changes in the brain? Mol Vis 18:2700-2710

20. Hodges JR et al (2004) Clinicopathological correlates in frontotemporal dementia. Ann Neurol 56(3):399-406

21. Jackson GR, Owsley C (2003) Visual dysfunction, neurodegenerative diseases, and aging. Neurol Clin 21(3):709-728

22. Jorge Cardoso M et al (2013) STEPS: similarity and truth estimation for propagated segmentations and its application to hippocampal segmentation and brain parcelation. Med Image Anal 17(6):671-684

23. Kim BJ et al (2017) Optical coherence tomography identifies outer retina thinning in frontotemporal degeneration. Neurology 89(15):1604-1611

24. Leger $\mathrm{F}$ et al (2011) Protein aggregation in the aging retina. J Neuropathol Exp Neurol 70(1):63-68

25. Ma D et al (2014) Automatic structural Parcellation of mouse brain MRI using multi-atlas label fusion. PLoS One 9(1):e86576

26. Ma Y et al (2008) In vivo 3D digital atlas database of the adult C57BL/6 mouse brain by magnetic resonance microscopy. Front Neuroanat 2(1)

27. Mazzaro N et al (2016) Tau-driven neuronal and neurotrophic dysfunction in a mouse model of early Tauopathy. J Neurosci 36(7):2086

28. Mendez MF, Cherrier MM, Meadows RS (1996) Depth perception in Alzheimer's disease. Percept Mot Skills 83(3):987-995

29. Miot-Noirault E et al (1997) T2 relaxation time as a marker of brain myelination: experimental MR study in two neonatal animal models. $J$ Neurosci Methods 72(1):5-14

30. Modat $\mathrm{M}$ et al (2010) Fast free-form deformation using graphics processing units. Comput Methods Prog Biomed 98(3):278-284

31. Nucci C et al (2011) Glaucoma progression associated with altered cerebral spinal fluid levels of amyloid beta and tau proteins. Clin Exp Ophthalmol 39(3):279-281

32. O'Callaghan J et al (2014) Is your system calibrated? MRI gradient system calibration for pre-clinical, High-Resolution Imaging. PLOS ONE 9(5):e96568

33. O'Callaghan J et al (2017) Tissue magnetic susceptibility mapping as a marker of tau pathology in Alzheimer's disease. Neurolmage 159:334-345

34. Ochiishi T et al (1994) Immunohistochemical localization of Ca2+/calmodulindependent protein kinase II in the rat retina. Brain Res 634(2):257-265

35. Ou X et al (2009) Quantitative magnetization transfer measured Pool size ratio reflects optic nerve myelin content in ex vivo mice. Magn Reson Med 61(2):364-371

36. Ourselin S et al (2000) Block Matching: A General Framework to Improve Robustness of Rigid Registration of Medical Images. In: Delp SL, DiGoia AM, Jaramaz B (eds) Medical Image Computing and Computer-Assisted Intervention - MICCAI 2000: Third International Conference, Pittsburgh, PA, USA, October 11-14, 2000. Proceedings. Springer Berlin Heidelberg, Berlin, Heidelberg, pp 557-566

37. Pang J-J, Wu SM (2011) Morphology and immunoreactivity of Retrogradely double-labeled ganglion cells in the mouse retina. Invest Ophthalmol Vis Sci 52(7):4886-4896

38. Pérez De Sevilla Müller L, Shelley J, Weiler R (2007) Displaced amacrine cells of the mouse retina. J Comp Neurol 505(2):177-189
39. Rademakers R, Cruts M, van Broeckhoven C (2004) The role of tau (MAPT) in frontotemporal dementia and related tauopathies. Hum Mutat 24(4):277-295

40. Rascovsky K et al (2011) Sensitivity of revised diagnostic criteria for the behavioural variant of frontotemporal dementia. Brain 134(9):2456-2477

41. Rohrer JD et al (2011) Clinical and neuroanatomical signatures of tissue pathology in frontotemporal lobar degeneration. Brain 134(9):2565-2581

42. Sahara $\mathrm{N}$ et al (2014) Age-related decline in white matter integrity in a mouse model of tauopathy: an in vivo diffusion tensor magnetic resonance imaging study. Neurobiol Aging 35(6):1364-1374

43. SantaCruz K et al (2005) Tau suppression in a neurodegenerative mouse model improves memory function. Science 309(5733):476

44. Seelaar $\mathrm{H}$ et al (2011) Clinical, genetic and pathological heterogeneity of frontotemporal dementia: a review. J Neurol Neurosurg Psychiatry 82(5):476

45. Velasco A et al (2008) Detection of filamentous tau inclusions by the fluorescent Congo red derivative FSB [(trans,trans)-1-fluoro-2,5-bis(3hydroxycarbonyl-4-hydroxy)styrylbenzene]. FEBS Lett 582(6):901-906

46. Warren JD, Rohrer JD, Rossor MN (2013) Frontotemporal dementia. The BMJ 347:44827

47. Wells JA et al (2015) In vivo imaging of tau pathology using multiparametric quantitative MRI. Neurolmage 111:369-378

48. Yoneda $S$ et al (2005) Vitreous fluid levels of $\beta$-amyloid(1-42) and tau in patients with retinal diseases. Jpn J Ophthalmol 49(2):106-108

49. Yushkevich PA et al (2006) User-guided 3D active contour segmentation of anatomical structures: significantly improved efficiency and reliability. Neurolmage 31(3):1116-1128
Ready to submit your research? Choose BMC and benefit from:

- fast, convenient online submission

- thorough peer review by experienced researchers in your field

- rapid publication on acceptance

- support for research data, including large and complex data types

- gold Open Access which fosters wider collaboration and increased citations

- maximum visibility for your research: over $100 \mathrm{M}$ website views per year

At BMC, research is always in progress.

Learn more biomedcentral.com/submissions 